\title{
Study on the Strategy of Path Optimization on the Process of Air Handling Based on Genetic Algorithm
}

\author{
Jiwan Hu, Jianjian Li, Wude Xie, Qiang Peng \\ Unit 63981 of PLA, Wuhan, Hubei \\ E-mail: hujiwan110@163.com
}

Keywords: Air-conditioning unit; Psychrometric chart; Genetic algorithm; Optimal path

\begin{abstract}
With its great ability on controlling air parameters, combined air-conditioning unit is playing an increasingly important role in civilian life, industrial production and even military fields. Because of the variety of varied path, while the environment changed, the optimal varied path can't be got, and the unit can't be in the best operating state, which waste the energy serious. Through the analysis of the structure and principles of the unit, the paper aimed at studying the air condition on the psychrometric chart. And then use genetic algorithm to get the optimal value, finally the optimal varied path in the process of air treatment, which improving the efficiency of the unit greatly, and reducing the energy consumption.
\end{abstract}

\section{Air-conditioning Unit Structure}

The means that the unit treated air includes supplying air, filtrating, cooling, heating, dehumidifying, humidifying and so on. Different functional sections correspond to different devices, including forced draught fan, primary efficiency filter, electric heater, front surface air coolers, after surface air coolers, disc dehumidifier, and humidifier. The air-conditioning unit structure is shown in Figure 1.

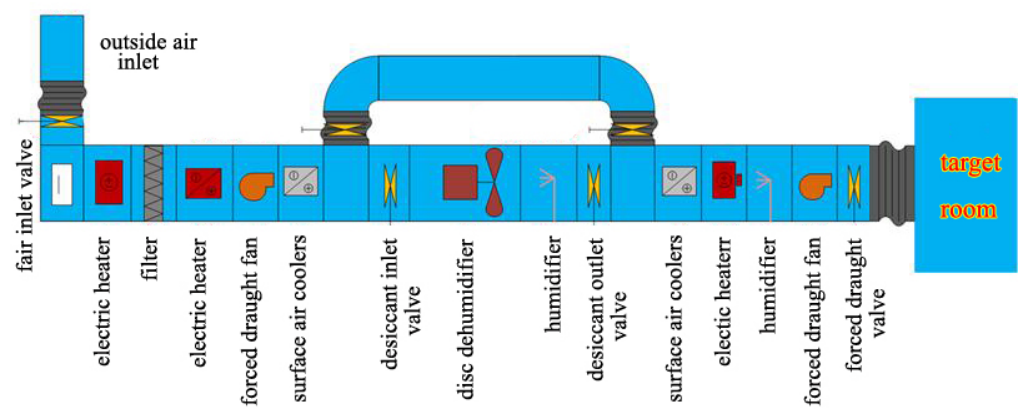

Figure 1 air-conditioning unit structure diagram

The corresponding functions of the main energy consumption equipment are shown below:

Forced draught fan: Transferring the outside air to the pipe of unit.

Primary efficiency filter: Filtering the dust in the air and improving the quality of the air.

Electric heater: Heating the air through the heat generated by the current through the resistance wire.

Surface air coolers: The purpose is cooling air through exchanging the heat and moisture and using the refrigerant as medium.

Disc dehumidifier: When the air through the dehumidification zone, the water is absorbed, so the effect of dehumidifying air is achieved.

Humidifier: The stainless steel electric heating pipe was used to heating the water in the tank, and then the steam was generated which would be sprayed into the air, so the air was cleared and humidified.

\section{Analysis on characteristics of operating conditions}

The change of air condition is a very complicated process, a single mathematical formula or a diagram is difficult to reflect the air handing process ${ }^{[2]}$. The state of the air is characterized by the 
psychrometric chart in engineering. Based on the calculation formula of enthalpy and moisture content of the moist air which is composited by $1 \mathrm{~kg}$ dry air under certain atmospheric pressure, the psychrometric chart was designed which toke the temperature as vertical coordinates and the moisture content as horizontal coordinates.

The means that different devices dispose air is varied. When through heater, the air is heated while the moisture content is unchanged. When through surface air coolers, the air is cooled while the moisture content is constant or the temperature and the moisture content of the air are both reduced. When through humidifier, the air is humidified while the temperature is constant. When through disc dehumidifier, the air is dehumidified while the enthalpy is constant. The changed process is reflected in psychrometric chart as shown in figure 3.

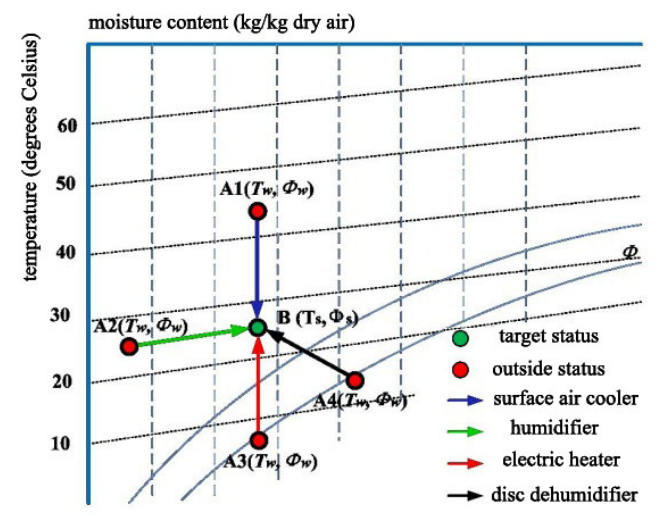

Figure 3 the process that devices dispose air

\section{Condition of high-temperature and high-humidity}

The operating condition of high-temperature and high-humidity is a more complicated condition. There are different ways when the status of air changes from high-temperature and high-humidity status to target status, but some ways is impossible in actual operation, so the air can only be cooled and dehumidified under this condition. The devices with cooling function includes front surface air coolers and after surface air coolers, with dehumidifying function includes disc dehumidifier, and the position of the three devices in the unit is: front surface air coolers, after surface air coolers and disc dehumidifier. Any combination of the three devices can achieve the change that the air changed from fresh status to target status.

When the three devices are all opened, the air is cooled while the moisture content is constant when through the front surface air cooler. Before the temperature drops to the dew point temperature, the disc dehumidifier begins working and the air is dehumidified while the enthalpy is constant. Because of the temperature rising in the dehumidifying process, the after surface air cooler should be opened to cool the air under constant moisture content, then the target temperature and humidity. The process that the air in the circumstances contains: supplying air, cooled under constant moisture content, dehumidified while the enthalpy is constant, cooled more, and supplying air, which is defined as situation1. The corresponding devices that are needed to open include: forced draught fan, front surface air coolers, disc dehumidifier, after surface air coolers, and forced draught fan, which is defined as combination 1.

When the front surface air cooler is closed, the process that the air in the circumstances contains: supplying air, dehumidified while the enthalpy is constant, cooled more, and supplying air, which is defined as situation 2. The corresponding devices that are needed to open include: forced draught fan, disc dehumidifier, after surface air coolers and forced draught fan, which is defined as combination 2.

When the after surface air cooler is closed, the process that the air in the circumstances contains: supplying air, cooled under constant moisture content and supplying air which is defined as situation 3. The corresponding devices that are needed to open include: forced draught fan, front surface air coolers, disc dehumidifier and forced draught fan, which is defined as combination 3.The change process is shown in figure 4. 


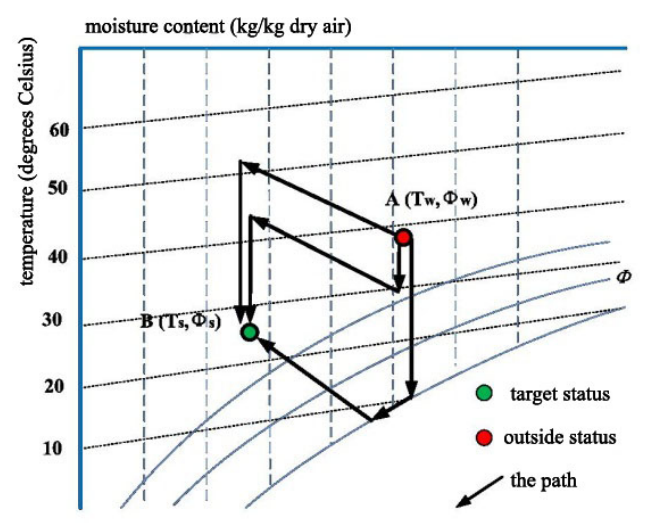

Figure 4 the path diagram of varied air status in high-temperature and high-humidity

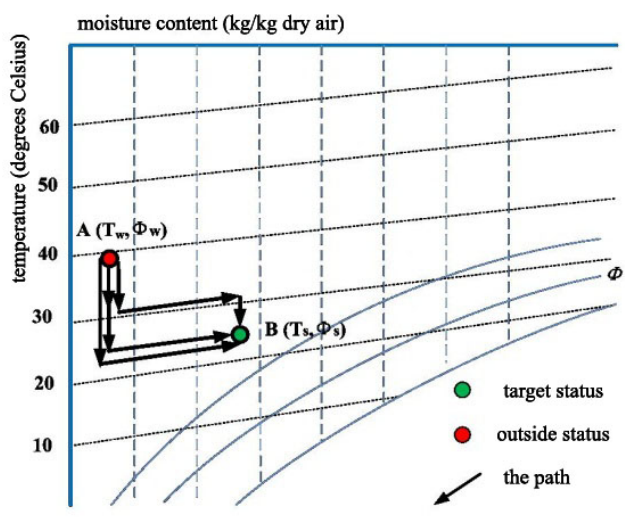

Figure 5 the path diagram of varied air status in high-temperature and low-humidity

From figure 4, we can know that the air in the varied process will experience multiple intermediate states points. The corresponding devices will consume a certain amount of electric energy when the air changed from one state point to another point, and the amount of energy consumption depends on the actual control parameters of the devices. so when the optimal intermediate state point is determined, the optimal control parameters in the actual operation will be determined too, which can ensure that the unit can work with the minimum energy consumption point.

\section{Condition of high-temperature and low-humidity}

Like with the analysis of the operating condition of high-temperature and high-humidity, when the air changed from the status of high-temperature and low-humidity, the air will be cooled and humidified. The devices with cooling function includes front surface air coolers and after surface air coolers, with humidifying function includes humidifier, and the position of the three devices in the unit is: front surface air coolers, after surface air coolers and humidifier. Any combination of the three devices can achieve the change that the air changed from fresh status to target status.

When the three devices are all opened, the process that the air in the circumstances contains: supplying air, cooled under constant moisture content, cooled more, humidified while the temperature is constant and supplying air, which is defined as situation 1 . The corresponding devices that are needed to open include: forced draught fan, front surface air coolers, after surface air coolers, humidifier and forced draught fan, which is defined as combination 1.

When the after surface air cooler is closed, the process that the air in the circumstances contains: supplying air, cooled under constant moisture content, humidified under constant temperature and supplying air which is defined as situation 2. The corresponding devices that are needed to open include: forced draught fan, front surface air coolers, humidifier and forced draught fan, which is defined as combination 2.

When the front surface air cooler is closed, the process that the air in the circumstances contain: supplying air, cooled under constant moisture content, humidified under constant temperature and supplying air which is defined as situation 3. The corresponding devices that are needed to open include: forced draught fan, after surface air coolers, humidifier and forced draught fan, which is defined as combination 3.The change process is shown in figure 5.

\section{Condition of low-temperature and low-humidity}

This condition is relatively unusual in actual environment. Like with the analysis of the operating condition of high-temperature and high-humidity, when the air changed from the status of low-temperature and low-humidity, the possible process includes:

Situation 1: supplying air, heated under constant moisture content, humidified under constant temperature, heated under constant moisture content and supplying air.

Situation 2: supplying air, heated under constant moisture content, humidified under constant temperature and supplying air.

The different ways of combination of the corresponding devices are exist:

Combination 1: Forced draught fan, heater, humidifier, heater, and forced draught fan; 
Combination 1: Forced draught fan, heater, humidifier, and forced draught fan.

The change process is shown in figure 6.

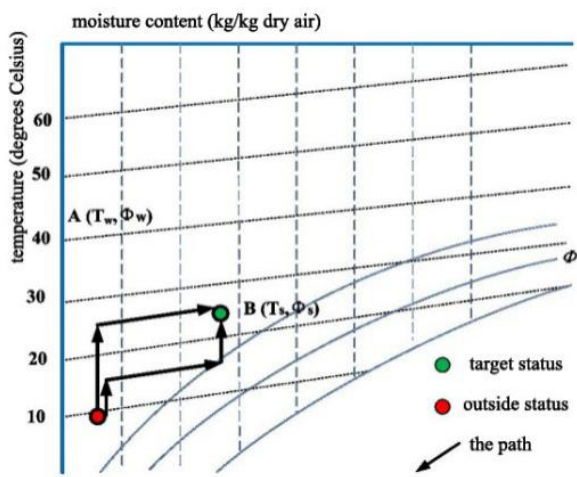

Figure 6 the path diagram of varied air status in low-temperature and low-humidity

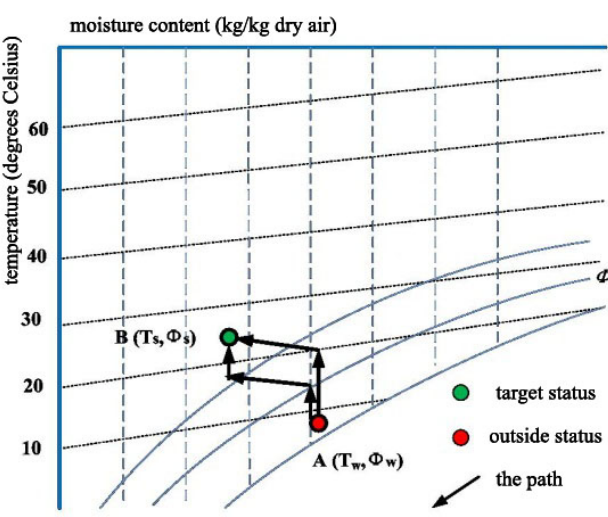

Figure 7 the path diagram of varied air status in low-temperature and high-humidity

\section{Condition of low-temperature and high-humidity}

Like with the analysis of the operating condition of high-temperature and high-humidity, when the air changed from the status of low-temperature and high-humidity, the possible process includes:

Situation 1: supplying air, heated under constant moisture content, dehumidified under constant enthalpy, heated under constant moisture content and supplying air.

Situation 2: supplying air, heated under constant moisture content, dehumidified under constant enthalpy and supplying air.

The different ways of combination of the corresponding devices are exist:

Combination 1: Forced draught fan, heater, disc dehumidifier, heater, and forced draught fan;

Combination 1: Forced draught fan, heater, disc dehumidifier, and forced draught fan.

The change process is shown in figure 7.

\section{Parameter Optimization}

The optimization research of the unit usually involves many problems such as multi variable, time variant, nonlinear and strong coupling. Traditional optimization algorithm cannot achieve the purpose of optimal control. While as a new kind of optimization algorithm, genetic algorithm can solve the above problems. The important calculation method to optimization research of unit is to global optimizing the control parameters in real time and online ${ }^{[3]}{ }^{[4]}$.The simulation model of genetic algorithm is based on MATLAB software, specific processes include:

\section{Determination of the length of binary string}

Binary coding is used as the coding method of the paper. Binary coding is one of the most frequently used methods. It is a set of symbols made up of binary symbols, whose length is related to the accuracy of the solution and the numbers of optimization parameters. Specific operations are as follows:

The parameters that represents in the range $[\mathrm{a}, \mathrm{b}]$ are represented as a binary string, the accuracy is precise to decimal places to $\mathrm{n}$. The closed interval $[\mathrm{a}, \mathrm{b}]$ is divided into $\left([b-a] \times 10^{n}\right)$ parts. The range of $[b-a] \times 10^{n}$ is determined.

$2^{m-1} \leq[b-a] \times 10^{n} \leq 2^{m}$

So the length of binary string needs at least $\mathrm{m}$.

\section{Parameter Selection}

Genetic algorithm is a calculation based on population. The size of population should not be too large and the general range is in 20 to 100. The size of population in the paper is 100. After repeater screening and verification, the crossover probability is determined as 0.7 and mutation probability is determined as 0.08 . The size of the number of iterations is corresponding to the speed and accuracy of solution. If the number of iterations is too small, it is easy to cause miscarriage of justice and the 
accuracy of the solution will be reduced. If the number of iterations is too large, the speed of solution will be reduced too and the computing cycle will be extended. Finally the number of iterations is determined as 100 by the collation and analysis of data.

\section{Determination of Objective Function}

The minimum value of the total energy consumption is optimized by taking the total energy consumption as objective function and taking the parameters that need to be optimized as arguments. The total energy corresponded to different combinations of devices is different. The respective model of objective function is established to analyze and simulate, and finally the optimal combinations is determined by comparing the total energy consumption.

\section{Binary Decoding}

The parameters that had been optimized need decoding calculation and then convert to decimal parameters.

For example, if a binary string such as $\left(b_{n} b_{n-1} \ldots b_{2} b_{1}\right)$ want to translate into the corresponding real value between $[\mathrm{a}, \mathrm{b}]$. Specific decoding operation is:

The binary numbers are represented as a decimal number:

$$
\left(b_{n} b_{n-1} \ldots b_{2} b_{1}\right)_{2}=\left(\sum_{l=1}^{n} b_{l} \cdot 2^{l}\right)_{10}=x
$$

Reals that corresponding to $\mathrm{x}$ ' range in $[\mathrm{a}, \mathrm{b}]$ is:

$$
x=-1.0+x^{\prime} \cdot \frac{b-a}{2^{n}-1}
$$

\section{Analysis of Result}

Combined with the actual environment, the parameters in actual operation is optimal analyzed under the condition of high-temperature and humidity and the condition of low-temperature and high-humidity, the optimization result is shown below.

\section{The optimization and analysis of the condition of high-temperature and humidity}

The selected temperature of the fresh air is 21.42 degrees Celsius and the moisture content is selected as 73.76 ( $\mathrm{kg} / \mathrm{kg}$ dry air). The target temperature is selected as 16.00 degrees Celsius and the target moisture content is selected as 30.00 (kg/kg dry air). The fan is input as variable frequency, and then the initial frequency is set as $35 \mathrm{HZ}$.

The three parameters corresponding to situation 1 are need to be optimized: the target temperature behind the front surface air cooler, the target moisture content behind disc dehumidifier and the target temperature behind the after surface air cooler. The optimization process of optimal energy consumption corresponding to situation 1 is shown in figure 8.

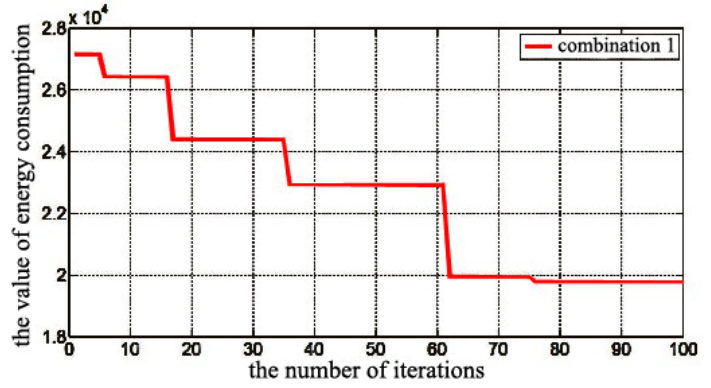

Figure 8 the relation between energy consumption value corresponding to situation 1 and iteration numbers

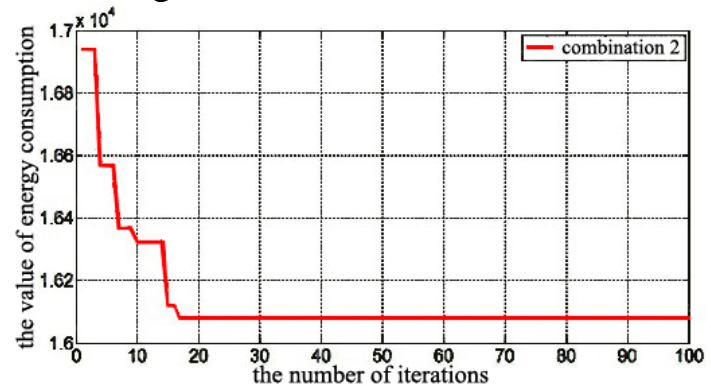

Figure 9 the relation between energy consumption value corresponding to situation 2 and iteration numbers

From figure 8, we can know that: after 76 iterations, the energy consumption of the system is achieved and is kept in $1.96 \times 10^{4} \mathrm{~kW}$. Then the optimal operation parameters are achieved while is existed as binary strings. So these parameters are needed to be recoded.

The length of binary string of single variables is determined as 10 through calculations, so the length of the whole string in situation 1 is 30 , which can be represented as $b_{29} b_{28} \ldots b_{1} b_{0}$. Among them, 
$b_{9} b_{8} \ldots b_{1} b_{0}$ is represented as the binary string of the target temperature behind the front surface air cooler, $b_{19} b 1_{8} \ldots b_{11} b_{10}$ is represented as the binary string of the target moisture content behind disc dehumidifier, $b_{29} b_{28} \ldots b_{21} b_{20}$ is represented as the binary string of the target temperature behind the after surface air cooler. Based on the decoding operation in the last section, the three binary strings are decoded.

First the binary numbers are converted to decimal numbers:

$$
\begin{aligned}
& \left(b_{9} b_{8} \ldots b_{1} b_{0}\right)_{2}=\left(\sum_{l=0}^{9} b^{l} \cdot 2^{l}\right)_{10}=x_{1}{ }^{\prime} \\
& \left(b_{19} b_{18} \ldots b_{11} b_{10}\right)_{2}=\left(\sum_{l=10}^{19} b^{l} \cdot 2^{l}\right)_{10}=x_{2}{ }^{\prime}\left(b_{29} b_{28} \ldots b_{21} b_{20}\right)_{2}=\left(\sum_{l=20}^{29} b^{l} \cdot 2^{l}\right)_{10}=x_{3}{ }^{\prime}
\end{aligned}
$$

Then $x_{1} 、 x_{2} 、 x_{3}$ are converted to real numbers in corresponding range:

$$
\begin{aligned}
& x_{1}=-1.0+x_{1} \cdot \frac{\left(t_{B . o}\right)_{\max }-\left(t_{B . o}\right)_{\min }}{2^{10}-1} \\
& x_{2}=-1.0+x_{2} \cdot \frac{\left(d_{Z . o}\right)_{\max }-\left(d_{Z . o}\right)_{\min }}{2^{10}-1} x_{3}=-1.0+x_{3}{ }^{\prime} \cdot \frac{\left(t_{H . o}\right)_{\max }-\left(t_{H . o}\right)_{\min }}{2^{10}-1}
\end{aligned}
$$

The three parameters corresponding to situation 2 are needed to be optimized: the target temperature behind disc dehumidifier, the target moisture content behind disc dehumidifier and the target temperature behind the after surface air cooler. The optimization process of optimal energy consumption corresponding to situation 2 is shown in figure 9.

From figure 9, we can know that: after 18 iterations, the energy consumption of the system is achieved and is kept in $1.61 \times 10^{4} \mathrm{~kW}$.

The three parameters corresponding to situation 3 are needed to be optimized: the target temperature behind the front surface air cooler, the target temperature behind disc dehumidifier and the target moisture content behind disc dehumidifier. The optimization process of optimal energy consumption corresponding to situation 2 is shown in figure 10.

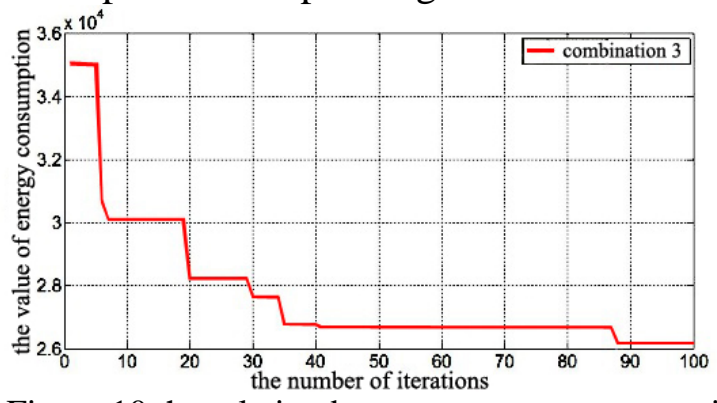

Figure 10 the relation between energy consumption value corresponding to situation 2 and iteration numbers

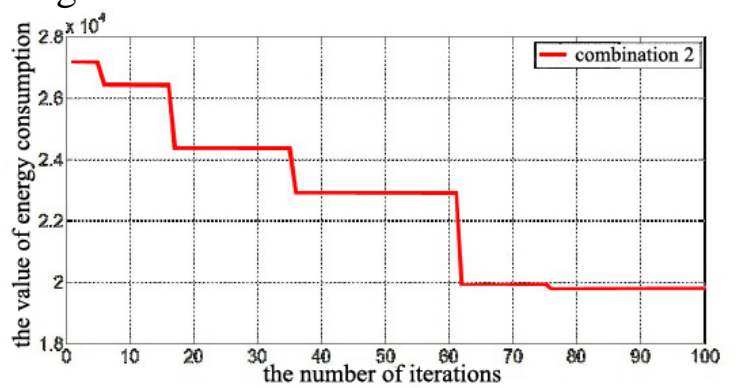

Figure 11 the relation between energy consumption value corresponding to situation 2 and iteration numbers

From figure 10, we can know that: after 88 iterations, the energy consumption of the system is achieved and is kept in $2.61 \times 10^{4} \mathrm{~kW}$, the target temperature behind the front surface air cooler is $19.32^{\circ} \mathrm{C}$, the target temperature behind disc dehumidifier is $16.05^{\circ} \mathrm{C}$, the target moisture content behind disc dehumidifier is $30.04(\mathrm{~kg} / \mathrm{kg}$ dry air).

By comparing the three values of energy consumption of three combinations, the value of energy consumption in combination 2 is the lowest, so the optimal operation state of unit is determined: forced draught fan, disc dehumidifier, after surface air coolers and forced draught fan.

\section{The optimization and analysis of the condition of low-temperature and high-humidity}

Combined with the study on the characteristics of the working conditions of the first section, we can know that there are three paths under the condition of low-temperature and high-humidity and three combinations. Like with the analysis of the condition of high-temperature and humidity, The selected temperature of the fresh air is 4.42 degrees Celsius and the moisture content is selected as 72.76 (kg/kg dry air). The target temperature is selected as 16.00 degrees Celsius and the target moisture content is selected as 35.00 (kg/kg dry air). Then the lowest value of energy consumption is took as objective function and the constraint conditions of influence factors of energy 
consumption is determined, then the operation parameters are optimized by genetic algorithm, and finally the optimal path under this condition is determined by comparing the value of energy consumption of the three combination: supplying air, heated under constant moisture content, dehumidified under constant enthalpy and supplying air, the combination of the corresponding devices are: forced draught fan, heater, disc dehumidifier, and forced draught fan.

The optimization process of optimal energy consumption corresponding to situation 2 is shown in figure 11.

From figure 11, we can know that: after 76 iterations, the energy consumption of the system is achieved and is kept in $1.98 \times 10^{4} \mathrm{~kW}$. The optimal operation parameters are determined: the target temperature under heater is $15.60^{\circ} \mathrm{C}$, the target temperature behind disc dehumidifier is $16.03^{\circ} \mathrm{C}$ and the target moisture content behind disc dehumidifier is $34.35(\mathrm{~kg} / \mathrm{kg}$ dry air $)$.

\section{Conclusion}

Analysis of the above results showed that: the strategy of path optimization on the process of air handing based on genetic algorithm can optimize the path of air handing in real time and online, which can keep the operation parameter in optimal point of the status, and the control cycle would be reduced greatly, the operation efficiency would be improved greatly and the energy consumption would be reduced effectively.

\section{Reference}

[1] Zhao Rongyi, Fan Cunyang, Xue Dianhua etc. Air Conditioning [M]. Beijing: China Building Industry Press, 2009

[2] Wu Zejiang. Psychrometric calculation sheets for air handing process [J]. HV\&AC, 2011, 41(2):48-50.

[3] Zhou Zhi, Gan Shuchuan. Study on Optimization of Control Parameters Based on Genetic Algorithm [J]. Computer Applications: 2007, 27: 191-193.

[4] Zhao Yanfa, Wang Ruihua, Wang Pu. The Application of Genetic Algorithm in the Energy Optimization Control of the VAV system [J]. Control Engineering, 2009, 16(S1): 110-114

[5] Lei Yingjie. The MATLAB Toolbox and Application Based on Genetic Algorithm [M]. Xi'an: Xi'an Electronic Sience \& Technology University Press, 2005.

[6] Tang Juan. Simulation Analysis of Energy Consumption Model of Air Conditioning System Based on MATLAB Simulink [J]. Building Energy \& Environment, 2010, 29(2): 53-57. 